\title{
Exercise Sciences in the Aging World
}

\section{Cédric T Albinet ${ }^{\star}$}

CerCA - CNRS - Faculty of Sport Sciences, University of Poitiers, France

In 2010, WHO edited the "Global Recommendations on Physical Activity for Health" for various age groups. In adults aged 65 and older, WHO recommended that older adults should do at least 150 minutes of moderate-intensity aerobic physical activity (PA) or 75 minutes of vigorous-intensity PA per week, or any equivalent combination. Musclestrengthening activities and exercise to enhance balance and prevent falls should also be done two to three times a week. For additional health benefits, WHO recommended to double the dose of aerobic PA. Based on the scientific evidence available, these recommendations are thought to improve cardiorespiratory and muscular fitness, bone and functional health, and to reduce the risk of noncommunicable diseases, depression and cognitive decline. This evidence came from many studies in the field of exercise sciences, investigating, often independently, the physiological, psychological, cognitive, biomechanical, social or medical benefits of regular PA. Understanding and following those recommendations is not obvious however, and open questions still remain, as I will briefly exemplify below.

Although the definitions and the different ways to objectively or subjectively identify categories of exercise intensity is well accepted [1], their application to the older population is poorly understood and understudied. For instance, a moderate-intensity PA is defined as ranging from 3-6 METs (Metabolic EquivalenT; $1 \mathrm{MET}=3.5 \mathrm{~mL} \mathrm{O}_{2} /$ $\mathrm{kg} / \mathrm{min}$ ) or to have a relative intensity of $55<70 \% \mathrm{HRmax}$ (maximal heart rate). Common examples of moderate-intensity PAs are brisk walking, gentle swimming, double tennis or golf. A compendium of PA intensity (MET values) classifying the energy costs of hundred of PA, was developed by Ainsworth and collaborators in 1993 and updated in 2000 and 2011 [2]. However those reported values, based on normal adults' measures, have been recently challenged by Hall et al. [3], who showed that they may underestimate actual PA intensities for older adults aged 65 and older, maybe in part because their resting metabolic rate could be lower than $3.5 \mathrm{~mL} \mathrm{O} / \mathrm{kg} / \mathrm{min}$. Moreover, considering an absolute intensity of 3-6 METs to be a moderate-intensity PA without considering the individual aerobic fitness level $\left(\mathrm{VO}_{2} \max \right)$ may cause misinterpretations of actual energy expenditures. For instance, doing water aerobics (5.5 METs) for a 75 year old woman whose VO2max level is $20 \mathrm{~mL} \mathrm{O} / \mathrm{kg} / \mathrm{min}$ (5.7 METS) will not be experienced as a moderate-intensity PA, but will represent nearly her maximal intensity level. This simple example illustrates the need to use relative or subjective measures of intensity category for exercise training design and underscores the importance of conducting validation studies in the field of exercise physiology in older adults.

At a more fundamental level, several studies in the area of exercise sciences investigated the effects of regular PA on more basic specific functions or processes, such as aerobic and muscular fitness, balance and falls prevention, or cognitive vitality and brain health. These studies tended to define the best regiments of PA programs to exhibit specific improvements and to establish, where possible, dose-response relationships. Inspection of their conclusions show a great qualitative and quantitative heterogeneity of recommended PAs for expected specific benefits. The nature and quantity of exercises seem to differ depending on the principal outcome measure, though WHO recommendations broadly encompass all of them. Even in the same research domain, some discrepancies are noticeable. For instance, research in the recent but promising area of exercise effects on brain structure and function does not provide unequivocal conclusions. Authoritative and often cited studies reported that walking three times a week for at least six to twelve months can lead to great benefits in executive functions performance and structural and functional brain improvements [4-6], whereas other studies controlling more rigorously PA intensity levels or aerobic fitness improvements failed to show such positive relationship $[7,8]$. According to many researchers, aerobic exercise, at least at a moderateintensity level, is necessary (often sufficient) to obtain gains in cognitive and cerebral health, but others argue that resistance training at least two times a week can promote cognitive and functional brain plasticity in older adults [9]. An excellent recent review on this question argues that aerobic PA may have a different effect on cognitive and brain functions than coordinative PA [10]. Moreover, this review highlights the need to conduct studies testing directly the causal relationship between different PAs, brain changes and cognitive performance. The underlying cellular and molecular mechanisms of this causal relationship are not well understood and such studies are still too sparse. Recently, Erickson et al. [5] reported that a one-year moderate-intensity aerobic exercise program three days/week significantly increased hippocampal volume by $2 \%$ and improved spatial memory in adults aged 55-80 years. Another group who participated in a stretching and toning program experienced a $1.4 \%$ decline in hippocampal volume during the same period, but showed the same improvements in memory scores. The authors claimed that level of aerobic fitness could mediate the relationship between hippocampal volume and memory performance. These interesting results however leave open the question of causality because, if true, the stretching and toning group should have shown memory decline because of reduction in hippocampal volume. The clinical relevance of these results is also challenged by other results showing a substantial $6 \%$ grey matter volume reduction in middle-aged ultramarathon runners during the two-month Trans-Europe foot race [11]. Although not specifically studied, there was no reported cognitive deficit along this grey matter reduction. This process was shown to be reversible because eight months after the race, global brain volume came back to baseline level without new brain lesion. Collectively, this kind of result questions the exact mechanisms responsible for this brain plasticity, the dose-response relationship, but also the behavioral consequences of these changes.

Finally, as I pointed out in the introduction, following WHO recommendations on PA appears difficult considering about 30 to $50 \%$ of the population does not follow them and this prevalence is often

*Corresponding author: Cédric T Albinet, CerCA - CNRS - Faculty of Sport Sciences, University of Poitiers, France, Tel: 33 (0)5 494546 96; E-mail: cedric.albinet@univ-poitiers.fr

Received November 30, 2013; Accepted December 02, 2013; Published December 06, 2013

Citation: Albinet CT (2013) Exercise Sciences in the Aging World. Aging Sci 1: e107. doi: 10.4172/2329-8847.1000e107

Copyright: (c) 2013 Albinet CT. This is an open-access article distributed under the terms of the Creative Commons Attribution License, which permits unrestricted use, distribution, and reproduction in any medium, provided the original author and source are credited. 
more important within the elderly. Everyone knows that exercise is good for health but does not practice at the recommended level for health. Another challenge of exercise sciences in the field of health psychology is to understand the barriers to PA and to develop efficient strategies for behavior changes and exercise adherence. Some trials and surveys are conducted worldwide and can now give important insights to better promote physically active behavior as well as advice or recommendations to public authorities. To increase our level of knowledge, the various fields of exercise sciences not only must be aware of what is done and found but should cooperate to develop cross-disciplinary research projects. As a multi-disciplinary forum for the study of aging, the Journal of Aging Science and its readership are directly interested by these questions and are hopefully aware of the importance to develop real interdisciplinary research to investigate and resolve some of the above detailed open questions.

\section{References}

1. Norton K, Norton L, Sadgrove D (2010) Position statement on physical activity and exercise intensity terminology. J Sci Med Sport 13: 496-502.

2. Ainsworth BE, WL Haskell, Herrmann SD, Meckes N, Bassett DR, et al. (2011) 2011 Compendium of Physical Activities: a second update of codes and MET values. Med Sci Sports Exerc 43:1575-1581.

3. Hall KS, Howe CA, Rana SR, Martin CL, Morey MC (2013) METs and accelerometry of walking in older adults: standard versus measured energy cost. Med Sci Sports Exerc 45: 574-582.
4. Colcombe SJ, Kramer AF, Erickson KI, Scalf P, McAuley E, et al. (2004) Cardiovascular fitness, cortical plasticity, and aging. Proc Natl Acad Sci U S A 101: 3316-3321.

5. Erickson KI, Voss MW, Prakash RS, Basak C, Szabo A, et al. (2011) Exercise training increases size of hippocampus and improves memory: Proceedings of the National Academy of Sciences of the United States of America 108: 30173022.

6. Kramer AF, Hahn S, Cohen NJ, Banich MT, McAuley E, et al. (1999) Ageing fitness and neurocognitive function. Nature 400: 418-419.

7. Etnier JL, Nowell PM, Landers DM, Sibley BA (2006) A meta-regression to examine the relationship between aerobic fitness and cognitive performance. Brain Res Rev 52: 119-130.

8. Hoffman BM, Blumenthal JA, Babyak MA, Smith PJ, Rogers SD, et al. (2008) Exercise fails to improve neurocognition in depressed middle-aged and older adults. Med Sci Sports Exerc 40: 1344-1352.

9. Liu-Ambrose T, Nagamatsu LS, Voss MW, Khan KM, Handy TC (2012) Resistance training and functional plasticity of the aging brain: a 12-month randomized controlled trial. Neurobiol Aging 33: 1690-1698.

10. Voelcker-Rehage C, Niemann C (2013) Structural and functional brain changes related to different types of physical activity across the life span. Neurosci Biobehav Rev 37: 2268-2295

11. Freund W, Faust S, Birklein F, Gaser C, Wunderlich AP, et al. (2012) Substantial and reversible brain gray matter reduction but no acute brain lesions in ultramarathon runners: experience from the TransEurope-FootRace Project. BMC Med 10: 170 